Check for updates

Cite this: Chem. Sci., 2019, 10, 11103

๑ All publication charges for this article have been paid for by the Royal Society of Chemistry

Received 20th June 2019

Accepted 15th October 2019

DOI: $10.1039 /$ c9sc03040a

rsc.li/chemical-science

\section{Strong dual emission in covalent organic frameworks induced by ESIPT $\dagger$}

\author{
Hua-Qing Yin, Fangfei Yin and Xue-Bo Yin (D)*
}

Here we reveal the effects of hydrogen bonds and alkyl groups on the structure and emission of covalent organic frameworks (COFs). Hydrogen bonds improve molecular rigidity leading to high crystallinity and restrict intramolecular rotation to enhance the emission of COFs. An excited-state intramolecular proton transfer (ESIPT) effect for dual emission is achieved via the intramolecular hydrogen bonds between hydroxyl groups and imine bonds. Alkyl groups increase interlayer spacing as a natural "scaffold" and achieve a staggered $A B$ stacking mode to decrease aggregation-caused quenching. Based on the above guidance, $\mathrm{COF}-4-\mathrm{OH}$ with strong emission is prepared with 2,4,6-triformylphloroglucinol (TFP) and 9,9dibutyl-2,7-diaminofluorene (DDAF). Strong dual emission is observed and used to differentiate organic solvents with different polarities, to determine the water content in organic solvents, and to detect different $\mathrm{pH}$ levels. Our work serves as a guide for the rational design of functional monomers for the preparation of emissive COFs.

\section{Introduction}

Covalent organic frameworks (COFs) are a class of porous polymers prepared from organic building blocks with designable structures. ${ }^{1}$ Abundant monomers enable the realization of a wide range of applications of COFs. ${ }^{2-6}$ The properties of COFs can be designed and regulated at the atomic level by selecting appropriate monomers. ${ }^{7-9}$ Thus, COFs are expected to be supplementary molecular luminophores owing to their natural $\pi$-conjugated structure. Chromophore and aggregationinduced emission (AIE) cores have been used to prepare emissive COFs. ${ }^{10-16}$ 3-Dimensional COFs are a good platform for the preparation of emissive COFs, but the available molecular building blocks are limited. ${ }^{10,17}$ The main obstacles of fluorescent 2-dimensional COFs are non-radiative relaxation induced from intramolecular rotation and $\pi-\pi$ stacking selfquenching. ${ }^{18}$

Hydrogen bonds can enhance the emission of COFs by restriction of the intramolecular rotation (RIR). 2,4Dihydroxybenzene-1,3,5-tricarbaldehyde and anthracene-2,6diamine were used to prepare emissive COFs by the formation of intramolecular hydrogen bonds. ${ }^{19}$ The emission was improved for the COFs prepared with 1,6-diaminopyrene and 1,3,5-triformilfloroglucionol with intramolecular hydrogen bonds for RIR. ${ }^{8}$ Interlayer and intralayer hydrogen bonds were

State Key Laboratory of Medicinal Chemical Biology, Tianjin Key Laboratory of Biosensing and Molecular Recognition, College of Chemistry, Nankai University, Tianjin 300071, P. R. China

$\dagger$ Electronic supplementary information (ESI) available: Synthesis details and additional figures. See DOI: 10.1039/c9sc03040a confirmed to restrict intramolecular rotation with an eclipsed stacking mode to enhance the emission of COFs. ${ }^{20}$

Aggregation-caused quenching (ACQ) is another factor that disturbs the emission of COFs because of the $\pi-\pi$ stacking selfquenching. Mechanical exfoliation of nanosheets improves the emission of COFs by reducing $\pi-\pi$ stacking, but it is energyand time-consuming. ${ }^{12,21}$ Fluorescent COF nanosheets have been obtained through self-exfoliation. ${ }^{22}$ Steric hindrance of the side chains in monomers reduces the $\pi-\pi$ stacking and regulates the stacking mode of COFs to overcome ACQ. ${ }^{23-25}$ Pang et al. introduced ethyl or butyl groups into monomers to prepare COFs from 2,5-diethoxyterephthalaldehyde and $4,4^{\prime}, 4^{\prime \prime}, 4^{\prime \prime \prime}$-(ethene-1,1,2,2-tetrayl)tetraaniline. ${ }^{26} \mathrm{~A}$ different COF topology was realized. The various topologies and stacking modes may regulate the $\pi-\pi$ stacking to improve the emission, but the authors did not study the emission properties of their COFs.

Guidance on how to decrease intramolecular rotation and ACQ for emissive COFs is still limited. Intramolecular rotation of COFs increases thermal decay, which leads to a low quantum yield..$^{27}$ Large interlayer spacing decreases ACQ but increases molecular rotation; thus, ACQ and intramolecular rotation are a dilemma. Intramolecular hydrogen bonds increase molecular rigidity and decrease intramolecular rotation. ${ }^{28}$ The interlayer spacing and stacking mode govern the interlayer interaction. ${ }^{29}$ Thus, intramolecular rotation, interlayer spacing, and stacking mode are the critical keys for the emission of COFs. In addition to emission intensity, multiple emission and a large Stokes shift are also attractive for real applications..$^{30-32}$ Multiple emission enables ratiometric fluorescence sensing and visual 
detection. $^{33,34}$ A large Stokes shift decreases the interference from the excitation light.

Herein, we reveal the role of hydroxyl and alkyl groups in the preparation of emissive COFs with careful design of monomers. As the optimal structure, $\mathrm{COF}-4-\mathrm{OH}$ ("4" and "OH" represent $n$ butyl and hydroxyl groups, respectively) was prepared with 2,4,6-triformylphloroglucinol (TFP) and 9,9-dibutyl-2,7diaminofluorene (DDAF) through the Schiff base reaction. Intramolecular hydrogen bonds form between hydroxyl groups and imine nitrogen to induce RIR. Moreover, the hydrogen bond achieves excited-state intramolecular proton transfer (ESIPT) for dual emission at 400 and $590 \mathrm{~nm}$ under single excitation at $280 \mathrm{~nm}$ with a large Stokes shift. The $n$-butyl groups act as a natural "scaffold" to increase the interlayer spacing and achieve a staggered $\mathrm{AB}$ mode to decrease the interlayer stacking in traditional COFs. The dilemmatic ACQ and intramolecular rotation are simultaneously eliminated. The strong dual emission, in combination with multi-active sites, such as the hydroxyl, alkyl, and imine groups in $\mathrm{COF}-4-\mathrm{OH}$, is used to differentiate solvents with different polarities, to determine the water content in organic solvents, and to detect $\mathrm{pH}$. We trust that the results could serve as guidance for preparing fluorescent COFs with strong dual emission by the rational introduction of functional groups into the monomers.

\section{Results and discussion}

\section{COFs design to reveal the effect of hydroxyl and alkyl groups}

How to obtain highly fluorescent COFs still lacks guidance. The introduction of hydroxyl groups into the precursors is simple and practical for the preparation of emissive COFs. ${ }^{\mathbf{8 , 1 9 0} 20}$ Alkyl groups with different sizes influence the stacking mode and interlayer spacing of COFs to regulate their luminescence. Thus, TFP and 1,3,5-triformylbenzene (TFB) without hydroxyl groups are selected as aldehyde group-containing monomers. 2,7-Diaminofluorene (DAF) with two $\mathrm{R}$ groups $(\mathrm{R}=\mathrm{H}, n$-butyl, or $n$ octyl) on the same side is used as the amino-group-containing monomers (Fig. S1a $\dagger$ ). The Schiff base reaction between the aldehyde and amino groups enables the formation of different COFs (Fig. S1b $\dagger$ ). Therefore, the effect of hydroxyl and alkyl groups on the structure and emission of COFs is revealed (Scheme 1a). The $n$-butyl groups overcome ACQ, while -OH groups are used to form hydrogen bonds with imine nitrogen to induce RIR and realize ESIPT for dual emission under single excitation (Scheme 1b). Thus, strong dual emission is observed. The mechanism of ESIPT for dual emission is also revealed (Scheme 1c).

\section{Effects of hydroxyl and alkyl groups on the structure of COFs}

The effect of a hydroxyl group on the structure of COFs was firstly tested. COF-0 was prepared with DAF and TFB as monomers (Fig. 1a), ${ }^{35}$ whereas COF-4 was prepared with TFB and DDAF as monomers (Fig. 1b). No characteristic peak was observed in their PXRD patterns (Fig. 1c) with a spherical structure (Fig. S2a and $\mathrm{b} \dagger$ ). As a comparison, TFP was selected to react with DDAF or DAF to prepare COF-4-OH or $\mathrm{COF}-0-\mathrm{OH}$,
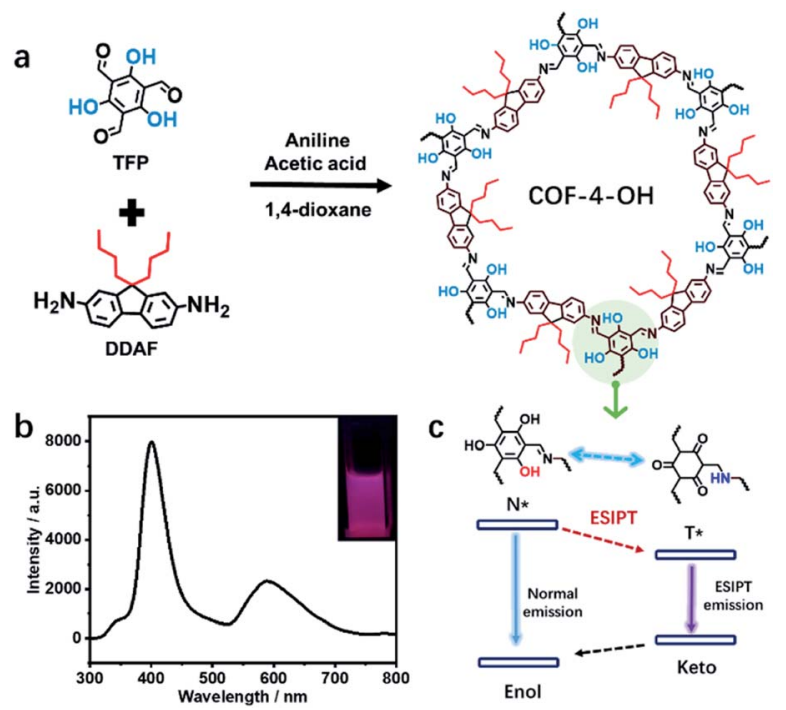

Scheme 1 (a) The design of COFs to reveal the effects of hydroxyl and alkyl groups and the ESIPT procedure, showing hydroxyl-functional TFP and alkyl groups-containing DDAF for the preparation of COF-4$\mathrm{OH}$. (b) Dual emission from COF-4-OH under $280 \mathrm{~nm}$ excitation; inset: a picture of COF-4-OH under $280 \mathrm{~nm}$ illumination. (c) The ESIPT dual emission mechanism of hydroxyl-containing COF-4-OH.

respectively (Fig. 1d and e). Characteristic peaks were observed from their PXRD patterns (Fig. 1f) to illustrate their higher crystallinity than COF-0 and COF-4. Intramolecular hydrogen bonds formed between imine nitrogen and hydroxyl groups, so intramolecular rotation decreased and the single layers of the COFs became rigid. The rigid layers stacked together regularly to produce crystalline COF-0-OH and COF-4$\mathrm{OH}$ with a rod-like structure (Fig. S2c and $\mathrm{d} \dagger$ ). Intramolecular hydrogen bonds act as a "lock" for RIR to achieve high crystallinity. The higher rigidity of the single layer may not result in a better PXRD pattern, but the good pattern clearly indicates that there is less intramolecular rotation. Thus, the intractable non-radiative decay induced by intramolecular rotation is overcome by the introduction of hydroxyl groups. However, interlayer stacking may occur, so stacking quenching or ACQ can still happen in COFs.

Alkyl groups with different lengths were tested to determine the interlayer spacing that can be utilized to overcome $\pi-\pi$ stacking. The following COFs with different alkyl groups were prepared: COF-0-OH, COF-4-OH, and COF-8-OH (where "0", “4”, and " 8 " represent no alkyl groups, $n$-butyl groups, and $n$-octyl groups, respectively). A gradual increase in the interlayer spacing was observed with 3.77, 5.53, and 6.42 A for COF-0-OH, COF-4-OH, and COF-8-OH, respectively (Fig. 2; more details are listed in Fig. S3-S5 $\dagger$ ). Generally, when the interlayer spacing is larger than $3.8 \AA$, the energy transfer from stacking interaction can be ignored. ${ }^{36,37}$ Therefore, ACQ from $\pi-\pi$ stacking is eliminated in COF-4-OH and COF-8-OH because the alkyl groups act as "scaffold" to prop up the layers. However, the interlayer spacing of the COFs increased nonlinearly. COF-4-OH with $n$ butyl groups showed a $1.76 \AA$ improvement over COF-0-OH, whereas a difference of $0.89 \AA$ was observed between COF-8- 

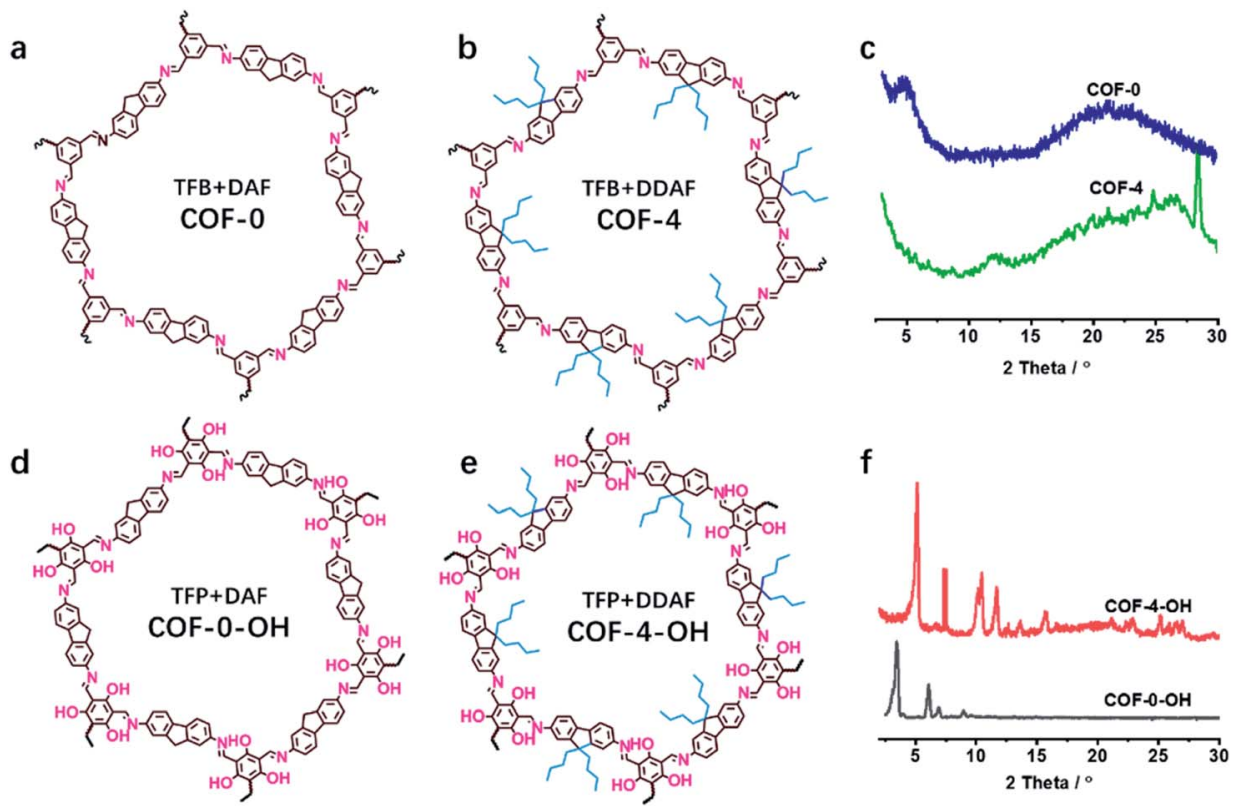

Fig. 1 The structures of (a) COF-O and (b) COF-4, and (c) their PXRD patterns. The structures of (d) COF-0-OH and (e) COF-4-OH, and (f) their PXRD patterns.

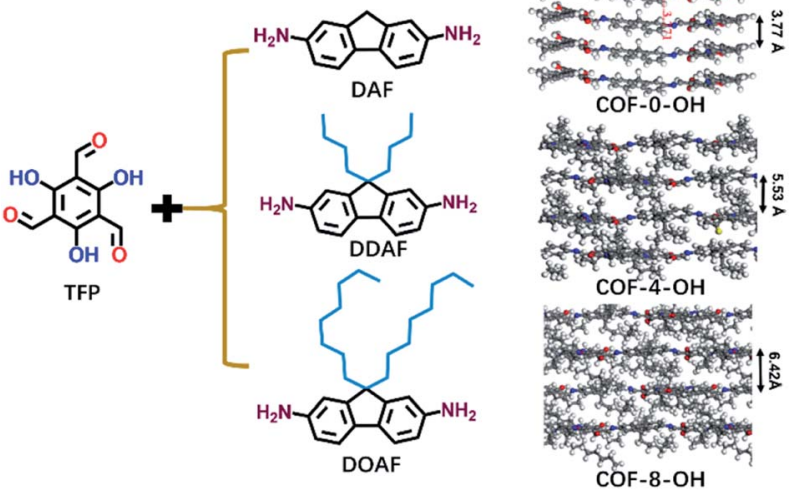

Fig. 2 The formation of COF-0-OH, COF-4-OH, and COF-8-OH to reveal the effects of alkyl groups on the interlayer spacings of different COFs.

$\mathrm{OH}$ and COF-4-OH. The enfoldment and twisting of long $n$-octyl groups are responsible for the low interlayer spacing improvement.

The stacking mode is another factor that influences the structure and fluorescence of COFs. The PXRD patterns were recorded to reveal the influence of the alkyl and -OH groups on the stacking mode and crystallinity of COFs. As shown in Fig. 3a, the $\mathrm{AB}$ model was consistent with the experimental PXRD pattern of COF-4-OH with an $R_{\mathrm{wp}}$ value of $9.45 \%$ and an $R_{\mathrm{p}}$ value of $6.53 \%$ after Pawley refinement. The strong PXRD peaks at $5.03^{\circ}, 7.62^{\circ}, 10.50^{\circ}$, and $11.68^{\circ}$ in COF-4-OH were assigned to the (100), (001), (300), and (220) facets, respectively (Fig. 3a). COF-0-OH exhibited a classical AA stacking with PXRD peaks at $3.45^{\circ}, 5.94^{\circ}$ and $6.89^{\circ}$ for the (100), (011), and (200) facets, respectively (Fig. 3b). Meanwhile, both COF-4-OH and COF-0-OH, with the help of $-\mathrm{OH}$ for the intramolecular hydrogen bonds, showed good PXRD patterns and thus high crystallinity. However, no obvious characteristic peaks were observed from the pattern of COF-8-OH because of the enfoldment and twisting of the long and soft $n$-octyl groups (Fig. S6 $\dagger$ ). Thus, overlong alkyl groups do not ensure the high crystallinity of COFs. COF- 4 with soft $n$-butyl groups also exhibited a poor PXRD pattern because of intramolecular rotation without the
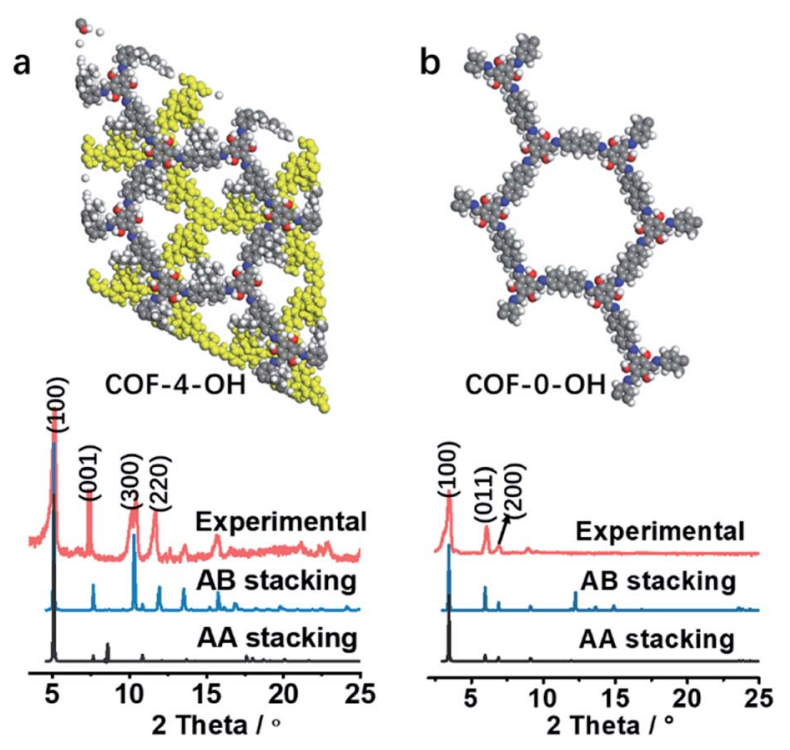

Fig. 3 (a) The experimental PXRD and refined PXRD patterns, and the $A B$ staggered stacking mode of COF-4-OH. (b) The experimental PXRD and refined PXRD patterns, and the AA eclipsed stacking mode of COF-O-OH. 
help of $-\mathrm{OH}$ (Fig. $\mathrm{S} 7 \dagger$ ). As a conclusion, $-\mathrm{OH}$ groups induce RIR to improve the rigidness of the single layer and thus realize COFs with high crystallinity. Larger alkyl groups result in a poorer PXRD pattern and lower crystallinity of the COFs because of the loose layer-by-layer stacking. Appropriate alkyl groups, such as $n$-butyl groups, allow COF-4-OH AB stacking.

\section{Characterization of COFs}

Fourier transform-infrared (FTIR) spectra were recorded to reveal the formation and structure of the COFs. For DDAF, the characteristic peak of $\mathrm{C}=\mathrm{N}$ appeared at $1614 \mathrm{~cm}^{-1}$ and $\mathrm{N}-\mathrm{H}$ peaks at about $3300 \mathrm{~cm}^{-1}$ disappeared to illustrate the occurrence of the Schiff base reaction (Fig. S8 $\dagger$ ). The $n$-butyl groups were observed in the FTIR spectra of COF-4-OH and DDAF. The peak of the imine bond was observed at $1614 \mathrm{~cm}^{-1}$ for both COF-4 and COF-0-OH (Fig. S9 and S10 $\dagger$ ). The carbon peak of $\mathrm{C}=\mathrm{N}$ was observed at $138 \mathrm{ppm}$ by ${ }^{13} \mathrm{C}$ solid-state nuclear magnetic resonance (NMR) spectroscopy, indicating the successful formation of the imine bond (Fig. S11†). Signals between 13 and $25 \mathrm{ppm}$ were related to the $n$-butyl groups in COF-4-OH and COF-4.

The keto and enol states were confirmed by FTIR and NMR spectra after COF-4-OH was soaked in methanol and $n$-hexane, respectively. The wide absorption around $3300-3700 \mathrm{~cm}^{-1}$ in the FTIR spectra was regarded as $-\mathrm{OH}$ groups for the enol state of $\mathrm{COF}-4-\mathrm{OH}$ in methanol; the characteristic peak of $\mathrm{C}=\mathrm{O}$ was clearly observed at $1715 \mathrm{~cm}^{-1}$ from COF-4-OH in hexane solution to reveal the existence of the keto state (Fig. S12 $\dagger$ ). The keto and enol states were also identified from the NMR spectra of $\mathrm{COF}-4-\mathrm{OH}$. The peak of $\mathrm{C}-\mathrm{OH}$ at $153 \mathrm{ppm}$ is related to the enol state and that of $\mathrm{C}=\mathrm{O}$ at $147 \mathrm{ppm}$ is observed for the keto state (Fig. S13†).

The permanent porosity and Brunauer-Emmett-Teller (BET) surface areas of $\mathrm{COF}-4-\mathrm{OH}$ and $\mathrm{COF}-0-\mathrm{OH}$ were characterized via $\mathrm{N}_{2}$ adsorption-desorption analysis at $77 \mathrm{~K}$ (Fig. S14 $\dagger$ ). The surface area of COF-4-OH was $34 \mathrm{~m}^{2} \mathrm{~g}^{-1}$ with a total pore volume of $0.11 \mathrm{~cm}^{3} \mathrm{~g}^{-1}$, whereas that of COF-0-OH was $339 \mathrm{~m}^{2}$ $\mathrm{g}^{-1}$ with a total pore volume of $0.50 \mathrm{~cm}^{3} \mathrm{~g}^{-1}$ at $P / P_{0}=0.99$. The alkyl groups reduced the pore volume and surface area of COF4-OH, so the porosity of COF-4-OH was lower than that of COF$0-\mathrm{OH}$. The BET specific surface area of COF-4-OH is quite low, which is similar to many other reported COFs with alkyl groups or guest molecules blocking their pores. ${ }^{24,35}$ The pore size of COF-4-OH is $5.0 \AA$ Abtained from BET analysis and is less than the simulated result of about $7.3 \AA$ obtained with Material Studio (Fig. S15 ${ }^{\dagger}$ ). As a comparison, the pore size of COF-0-OH obtained from the BET analysis and the simulation is $20.1 \AA$ and $27.7 \AA$, respectively (Fig. S16†). No remarkable weight loss was observed from COF-4-OH and COF-0-OH up to $380{ }^{\circ} \mathrm{C}$; meanwhile, COF- 4 was stable up to $300{ }^{\circ} \mathrm{C}$, as indicated by thermogravimetric analysis (Fig. S17-S19†). We also tested the chemical stability of COF-4-OH by dispersing it in tetrahydrofuran, water, ethanol, $\mathrm{HCl}(\mathrm{pH} 1)$, and $\mathrm{NaOH}(\mathrm{pH} 14)$ solutions at $25{ }^{\circ} \mathrm{C}$ for $24 \mathrm{~h}$. The characteristic absorption bands of $\mathrm{C}=\mathrm{N}$ were still observed in the FTIR spectra (Fig. S20†) so COF-4-OH was sufficiently stable for practical application.

\section{Optical properties}

The UV-vis absorption spectra of the monomers and COF-4-OH were investigated. TFP exhibited two absorption peaks at 270 and $370 \mathrm{~nm}$, whereas DDAF showed an absorption peak at $300 \mathrm{~nm}$ (Fig. S21 $\dagger$ ). After the formation of COF-4-OH, the main absorption band was red-shifted to $440 \mathrm{~nm}$ by the formation of the $\pi$-conjugated system. Intramolecular hydrogen bonds were evidenced by the shoulder peak at approximately $510 \mathrm{~nm}$. DDAF showed stronger fluorescence than DAF; meanwhile, the emission of TFP was red-shifted to $500 \mathrm{~nm}$ compared with that of TFB (Fig. S22 $\dagger$ ). COF-4-OH owns two emission bands at 400 and $590 \mathrm{~nm}$. While the emission at $590 \mathrm{~nm}$ was efficiently excited from 280 to $500 \mathrm{~nm}$, that at $400 \mathrm{~nm}$ was excited from 270 to $350 \mathrm{~nm}$ (Fig. 4a). On the basis of the 3D fluorescence mapping of COF-4-OH (Fig. S23 $\dagger$ ), under $280 \mathrm{~nm}$ excitation, equal dual emission was obtained. Hence, we selected $280 \mathrm{~nm}$ as the optimal excitation.

The luminescence of different COFs is recorded and discussed in terms of their structure, such as layer rigidity, interlayer spacing, and stacking mode. Nearly no emission was observed from COF-0 because of interlayer stacking and intramolecular rotation (Fig. 4b). Single emission was observed at $400 \mathrm{~nm}$ from COF-4 and the fluorescence quantum yield was about $0.1 \%$ (Fig. 4c). Although intramolecular rotation still exists, interlayer spacing is increased by the introduction of $n$ butyl groups, so the fluorescence is slightly enhanced for COF-4. After the introduction of hydroxyl groups, intramolecular hydrogen bonds formed, so dual emission with ESIPT effect is observed from COF-0-OH. However, the emission is weak with a quantum yield of $0.3 \%$ because of $\pi-\pi$ stacking (Fig. $4 d$ ). After hydroxyl groups and $n$-butyl were synergistically introduced, strong dual emission was observed from $\mathrm{COF}-4-\mathrm{OH}$ and the quantum yield increased to $10.7 \%$ (Fig. 4e). COF-8-OH exhibits dual emission but the intensity is weaker than that of $\mathrm{COF}-4-\mathrm{OH}$ with blue-shift (Fig. 4f). The low crystallinity of COF-8-OH is responsible for its low fluorescence intensity. The enfoldment and twisting of long and soft $n$-octyl groups led to irregular stacking in COF-8-OH. Thus, the non-radiative relaxation was enhanced, which decreased the emission of COF-8-OH.

Table S1† illustrates the emission wavelengths and quantum yields of different COFs. Fig. S24† provides the dual emission fluorescence profiles, where COF-4-OH showed the highest quantum yield. Two emissions were observed at 400 and $590 \mathrm{~nm}$ under single excitation of $280 \mathrm{~nm}$. The Stokes shifts were $120 \mathrm{~nm}\left(83333 \mathrm{~cm}^{-1}\right)$ and $310 \mathrm{~nm}\left(32258 \mathrm{~cm}^{-1}\right)$, respectively, to avoid interference from the excitation light. Dual emission with a wavelength difference of $190 \mathrm{~nm}\left(80075 \mathrm{~cm}^{-1}\right)$ is useful for ratiometric fluorescence sensing without interfering with each other. Thus, COF-4-OH was selected for further study in terms of its strong dual emission, large Stokes shift, and big gap between the two emissions.

\section{ESIPT mechanism}

The emission mechanism was explored using three molecular models (Fig. 5a and S25-27†). Model 1 showed dual emission to illustrate that both the alkyl and hydroxyl groups were essential 
a

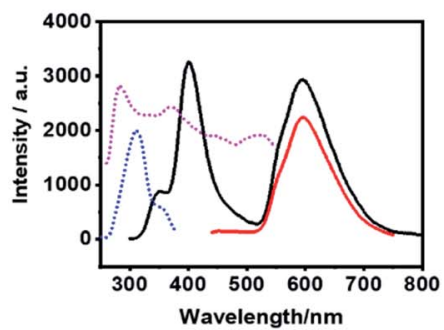

d

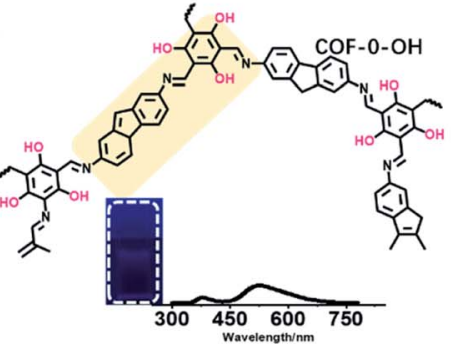

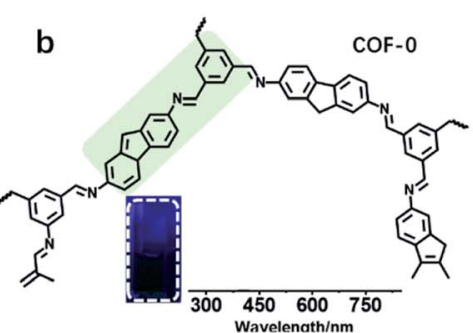

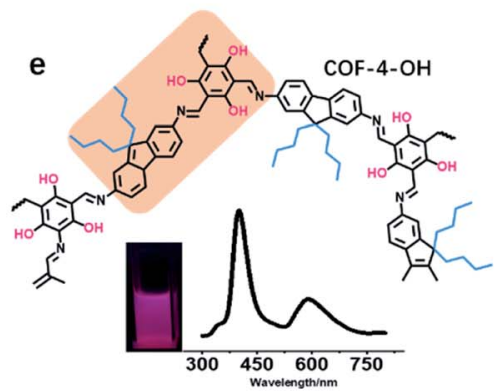

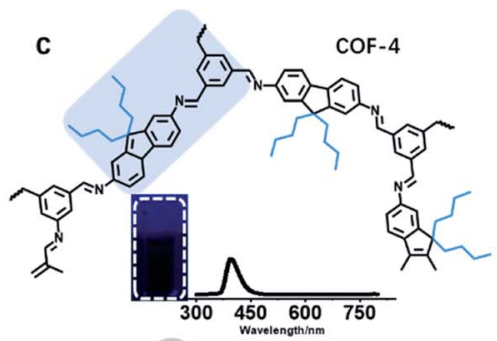

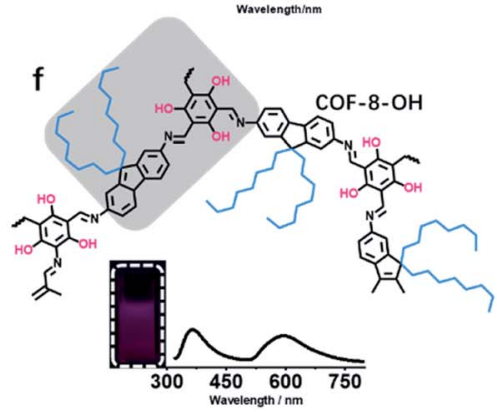

Fig. 4 The fluorescence properties of different COFs. (a) The fluorescence excitation and emission spectra of COF-4-OH; the dashed lines are excitation spectra; blue: $400 \mathrm{~nm}$ as emission; purple: $590 \mathrm{~nm}$ as emission; solid lines are emission spectra; black: under excitation of $280 \mathrm{~nm}$; red: under excitation of $400 \mathrm{~nm}$. Part structures of different COFs and pictures of COFs in DMF and their fluorescence profiles under $280 \mathrm{~nm}$ excitation: (b) COF-0, (c) COF-4, (d) $\mathrm{COF}-0-\mathrm{OH}$, (e) $\mathrm{COF}-4-\mathrm{OH}$, and (f) $\mathrm{COF}-8-\mathrm{OH}$.
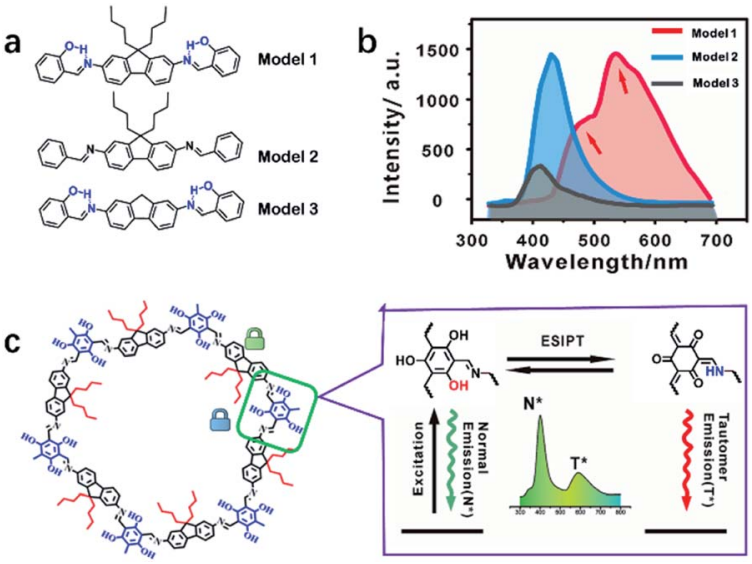

Fig. 5 (a) The structures of the three models: Model 1 vs. COF-4-OH, Model 2 vs. COF-4, and Model 3 vs. COF-O-OH. (b) Fluorescence spectra of the three models. (c) COF-4-OH fragment (left) and hydrogen bonding between the hydroxyl group and the nitrogen of imine realizes the ESIPT procedure for dual emission in COF-4-OH (right).

(Fig. 5b and S28†). Model 2 only exhibited single fluorescence because of the absence of an ESIPT structure without a hydroxyl group (Fig. S29†). Model 3 showed weak dual emission: the normal emission and the tail peak as ESIPT emission. Without the supporting effect of $n$-butyl groups, Model 3 molecules stack together and suffer from self-quenching for the lowest emission among the three models (Fig. 5b and S30 ${ }^{\circ}$ ). The emission centers and quantum yields of the models are listed in Table S1. $\uparrow$ COF-4-OH showed a larger wavelength gap than Model 1 because the $\pi$-conjugated system resulted in the two emissions shifting in opposite directions (Fig. S31†). ${ }^{38}$
Fig. 5c illustrates the ESIPT process for the dual emission of $\mathrm{COF}-4-\mathrm{OH}$. The blue emission at $400 \mathrm{~nm}$ was normal fluorescence from the enol state, whereas that at $590 \mathrm{~nm}$ with a larger Stokes shift was the ESIPT emission. The $n$-butyl groups and intramolecular hydrogen bonds act as dual locks for RIR to enhance the dual emission. The lifetime is $0.8 \mathrm{~ns}$ and $1.2 \mathrm{~ns}$ for the emissions from COF-4-OH at 400 and $590 \mathrm{~nm}$, respectively (Fig. S32 $\dagger$ ). The lifetime of the ESIPT emission is a little longer than that of the normal emission.

Time-dependent density functional theory (TD-DFT) calculation was used to determine the barrier gap between the enol and keto states (Fig. S33†). The barrier gap is $3.7 \mathrm{kcal} \mathrm{mol}^{-1}$, indicating that ESIPT happened in the COF-4-OH system. ${ }^{39}$ The strong dual emission was convenient for further use of COF-4$\mathrm{OH}$ as a probe because of the large Stokes shift and the big gap between the dual emissions.

The fluorescence profiles during the formation of $\mathrm{COF}-4-\mathrm{OH}$ were recorded to further validate the ESIPT effect. The intensity at about $410 \mathrm{~nm}$ decreased, whereas that at $550 \mathrm{~nm}$ increased gradually (Fig. S34†). Moreover, the emission at $410 \mathrm{~nm}$ blueshifted, whereas that at $550 \mathrm{~nm}$ red-shifted along with the reaction. The two emissions shifted in opposite directions after the formation of the $\pi$-conjugated system. It is an important property of ESIPT structures to validate the gradual formation of COF-4-OH. ESIPT emission occurs from intramolecular proton transfer in the excited state of COF-4-OH, so single excitation achieves dual emission and COF-4-OH is expected to be applied as a probe in ratiometric fluorescence sensing.

\section{Multi-target detection with $\mathrm{COF}-4-\mathrm{OH}$ as a probe}

Various active sites, such as imine, hydroxyl, and alkyl groups, exist in COF-4-OH and encourage us to explore multiple 
recognition. Water is often considered as a contaminant in organic solvents, and even trace amounts of water may cause failure in some chemical laboratories and industries. ${ }^{40}$ Thus, there is an urgent requirement to develop a simple water detection strategy. COF-4-OH is sensitive to water content in organic solvents with its intramolecular hydrogen bonds. The fluorescence intensity at $400 \mathrm{~nm}$ decreased, whereas that at $590 \mathrm{~nm}$ remained stable in ethanol with increased water content (Fig. 6a). The ratio of the two emissions decreased linearly for ratiometric sensing of water content (Fig. S35†). A color change from blue to red was clearly observed from the CIE chromaticity coordinates (inset in Fig. 6a). The limit of detection $(3 \sigma, v / v)$ for water content in ethanol was $0.03 \%$, which is equal to or less than that of other fluorescent strategies. ${ }^{\mathbf{2 8 , 4 1 , 4 2}}$ The dual emission response to water content in methanol, $N, N^{\prime}$ dimethylformamide, and dimethyl sulfoxide was used to validate the universality of COF-4-OH (Fig. S36†). Intermolecular hydrogen bonds form between hydroxyl groups and water molecules, so the structure of the enol form changes for less normal emission. However, the structure of the keto $\mathrm{C}=\mathrm{O}$ is relatively stable in aqueous conditions, so the ESIPT emission keeps stable.

ESIPT materials often show different fluorescence behaviours in different solvents, ${ }^{43}$ so we further used COF-4-OH as a probe to differentiate solvents with different polarities. As shown in Fig. 6b, the emission at $400 \mathrm{~nm}$ was enhanced in polar solvents, except for water, and the $590 \mathrm{~nm}$ emission decreased because less proton transfer occurs to transfer enol to keto forms in polar solvents. ${ }^{44}$ The emission intensity at different wavelengths was used for clustering analysis of the solvents. Obvious clustering was observed according to their polarity (Fig. 6c). Thus, COF-4-OH could be utilized to differentiate
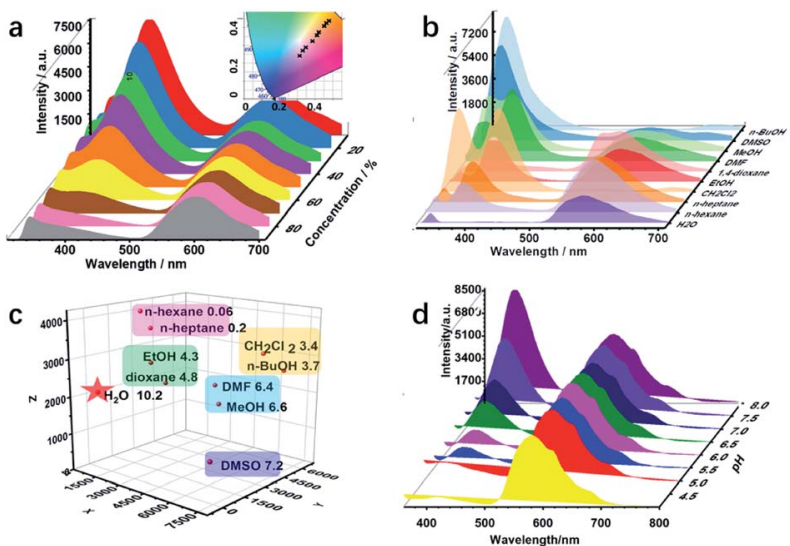

Fig. 6 (a) The fluorescence profiles of $\mathrm{COF}-4-\mathrm{OH}$ in ethanol with different water content from 10 to $90 \%$ (inset: CIE chromaticity coordinates of the emission from $\mathrm{COF}-4-\mathrm{OH}$ in response to the water content). (b) Fluorescence spectra of $\mathrm{COF}-4-\mathrm{OH}$ in solvents with different polarities. (c) 3-Dimensional coordinate fingerprint mapping of the solvents (the $X, Y$, and $Z$ axes represent the emission intensities of the peaks at about 335, 400, and $590 \mathrm{~nm}$ in (b); the polarities are shown behind the solvents). (d) Fluorescence response of COF-4-OH to different $\mathrm{pH}$ values. solvents with fingerprint mapping of fluorescence intensities and wavelengths.

COF-4-OH was also used to detect $\mathrm{pH}$ because of the existing intramolecular hydrogen bonds. As shown in Fig. 6d, the emission at $400 \mathrm{~nm}$ was obviously enhanced with the increase in $\mathrm{pH}$, whereas that at $590 \mathrm{~nm}$ stayed stable. Ratiometric sensing of $\mathrm{pH}$ was achieved; meanwhile, the CIE coordinates illustrated the visual detection of $\mathrm{pH}$ variation (Fig. S37†). We proposed that a hydrogen bond forms between the $-\mathrm{OH}$ group of the enol state and $\mathrm{H}^{+}$to form $-\mathrm{OH}_{2}{ }^{+}$, so the normal emission decreases in acid solution. With the increased $\mathrm{pH},-\mathrm{OH}_{2}{ }^{+}$ recovers to $-\mathrm{OH}$ and the normal emission is improved. The keto state of COF-4-OH was relatively stable for the invariable ESIPT emission.

\section{Conclusions}

In conclusion, the effects of the hydroxyl and alkyl groups in the monomers on the structure and emission of COFs were revealed. Correspondingly, COF-4-OH was prepared with strong dual emission and a large Stokes shift via the rational introduction of $n$-butyl and hydroxyl groups into the monomers. Intramolecular hydrogen bonding provides the ESIPT structure for dual emission. The large interlayer spacing, combined with the $\mathrm{AB}$ stacking mode, efficiently decreased thermal decay from $\pi-\pi$ stacking to enhance the emission. The $n$-butyl groups and hydrogen bonds restricted the intramolecular rotation synergistically. Thus, dilemmatic ACQ and intramolecular rotation were simultaneously eliminated to endow COF-4-OH with strong dual emission. The two emissions showed different colors, allowing ratiometric sensing for the differentiation of solvents of different polarities, the measuring of water content in organic solvents, and the determination of $\mathrm{pH}$ response. We provide guidance for the design of highly emissive COFs via the rational introduction of functional groups to overcome $\pi-\pi$ stacking and hydroxyl groups to decrease thermal decay from intramolecular rotation.

\section{Conflicts of interest}

There are no conflicts to declare.

\section{Acknowledgements}

This work was supported by the National Natural Science Foundation of China (Grants 21874074, 21675090, and 21435001) and the China Scholarship Council (20183101).

\section{References}

1 T. Ma, E. A. Kapustin, S. X. Yin, L. Liang, Z. Zhou, J. Niu, L. H. Li, Y. Wang, J. Su, J. Li, X. Wang, W. D. Wang, W. Wang, J. Sun and O. M. Yaghi, Science, 2018, 361, 48-52.

2 S. Kandambeth, K. Dey and R. Banerjee, J. Am. Chem. Soc., 2018, 141, 1807-1822.

3 J. L. Segura, M. J. Mancheno and F. Zamora, Chem. Soc. Rev., 2016, 45, 5635-5671. 
4 C. S. Diercks and O. M. Yaghi, Science, 2017, 355, 1-8.

5 A. P. Co, A. I. Benin, N. W. Ockwig, M. O'Keeffe, A. J. Matzger and O. M. Yaghi, Science, 2005, 310, 1166-1170.

6 G. H. Bertrand, V. K. Michaelis, T. C. Ong, R. G. Griffin and M. Dinca, Proc. Natl. Acad. Sci. U. S. A., 2013, 110, 4923-4928.

7 Z. Li, N. Huang, K. H. Lee, Y. Feng, S. Tao, Q. Jiang, Y. Nagao, S. Irle and D. Jiang, J. Am. Chem. Soc., 2018, 140, 1237412377.

8 P. Albacete, J. I. Martinez, X. Li, A. Lopez-Moreno, S. A. MenaHernando, A. E. Platero-Prats, C. Montoro, K. P. Loh, E. M. Perez and F. Zamora, J. Am. Chem. Soc., 2018, 140, 12922-12929.

9 E. Vitaku and W. R. Dichtel, J. Am. Chem. Soc., 2017, 139, 12911-12914.

10 H. Ding, J. Li, G. Xie, G. Lin, R. Chen, Z. Peng, C. Yang, B. Wang, J. Sun and C. Wang, Nat. Commun., 2018, 9, 5234. 11 S. Dalapati, E. Jin, M. Addicoat, T. Heine and D. Jiang, J. Am. Chem. Soc., 2016, 138, 5797-5800.

12 J. Dong, X. Li, K. Zhang, Y. Di Yuan, Y. Wang, L. Zhai, G. Liu, D. Yuan, J. Jiang and D. Zhao, J. Am. Chem. Soc., 2018, 140, 4035-4046.

13 S. Dalapati, S. Jin, J. Gao, Y. Xu, A. Nagai and D. Jiang, J. Am. Chem. Soc., 2013, 135, 17310-17313.

14 T. Y. Zhou, S. Q. Xu, Q. Wen, Z. F. Pang and X. Zhao, J. Am. Chem. Soc., 2014, 136, 15885-15888.

15 J. W. Crowe, L. A. Baldwin and P. L. McGrier, J. Am. Chem. Soc., 2016, 138, 10120-10123.

16 F. Auras, L. Ascherl, A. H. Hakimioun, J. T. Margraf, F. C. Hanusch, S. Reuter, D. Bessinger, M. Doblinger, C. Hettstedt, K. Karaghiosoff, S. Herbert, P. Knochel, T. Clark and T. Bein, J. Am. Chem. Soc., 2016, 138, 1670316710.

17 G. Lin, H. Ding, D. Yuan, B. Wang and C. Wang, J. Am. Chem. Soc., 2016, 138, 3302-3305.

18 M. R. Rao, Y. Fang, S. De Feyter and D. F. Perepichka, J. Am. Chem. Soc., 2017, 139, 2421-2427.

19 S. Haldar, D. Chakraborty, B. Roy, G. Banappanavar, K. Rinku, D. Mullangi, P. Hazra, D. Kabra and R. Vaidhyanathan, J. Am. Chem. Soc., 2018, 140, 1336713374.

20 X. Li, Q. Gao, J. Wang, Y. Chen, Z. H. Chen, H. S. Xu, W. Tang, K. Leng, G. H. Ning, J. Wu, Q. H. Xu, S. Y. Quek, Y. Lu and K. P. Loh, Nat. Commun., 2018, 9, 2335.

21 S. Wang, Q. Wang, P. Shao, Y. Han, X. Gao, L. Ma, S. Yuan, X. Ma, J. Zhou, X. Feng and B. Wang, J. Am. Chem. Soc., 2017, 139, 4258-4261.

22 A. Mal, R. K. Mishra, V. K. Praveen, M. A. Khayum, R. Banerjee and A. Ajayaghosh, Angew. Chem., Int. Ed., 2018, 130, 8579-8583.
23 S. Rager, A. C. Jakowetz, B. Gole, F. Beuerle, D. D. Medina and T. Bein, Chem. Mater., 2019, 31, 2707-2712.

24 Z. Li, Y. Zhang, H. Xia, Y. Mu and X. Liu, Chem. Commun., 2016, 52, 6613-6616.

25 N. Keller, D. Bessinge, S. Reuter, M. Calik, L. Ascherl, F. C. Hanusch, F. Auras and T. Bein, J. Am. Chem. Soc., 2017, 139, 8194-8199.

26 Z.-F. Pang, T.-Y. Zhou, R.-R. Liang, Q.-Y. Qi and X. Zhao, Chem. Sci., 2017, 8, 3866-3870.

27 Q. Gao, X. Li, G. H. Ning, K. Leng, B. Tian, C. Liu, W. Tang, H. S. Xu and K. P. Loh, Chem. Commun., 2018, 54, 2349-2352.

28 H. L. Qian, C. Dai, C. X. Yang and X. P. Yan, ACS Appl. Mater. Interfaces, 2017, 9, 24999-25005.

29 X. Wu, X. Han, Y. Liu, Y. Liu and Y. Cui, J. Am. Chem. Soc., 2018, 140, 16124-16133.

30 Z.-R. Yang, M.-M. Wang, X.-S. Wang and X.-B. Yin, Anal. Chem., 2017, 89, 1930-1936.

31 H. Q. Yin, J. C. Yang and X. B. Yin, Anal. Chem., 2017, 89, 13434-13440.

32 A. N. Butkevich, G. Lukinavicius, E. D'Este and S. W. Hell, J. Am. Chem. Soc., 2017, 139, 12378-12381.

33 M. H. Lee, J. S. Kim and J. L. Sessler, Chem. Soc. Rev., 2015, 44, 4185-4191.

34 N. Jiang, J. Fan, F. Xu, X. Peng, H. Mu, J. Wang and X. Xiong, Angew. Chem., Int. Ed., 2015, 54, 2510-2514.

35 L. Wang, B. Dong, R. Ge, F. Jiang and J. Xu, ACS Appl. Mater. Interfaces, 2017, 9, 7108-7114.

36 C. Janiak, J. Chem. Soc., Dalton Trans., 2000, 2000, 38853896.

37 K. Li, Y. Liu, Y. Li, Q. Feng, H. Hou and B. Z. Tang, Chem. Sci., 2017, 8, 7258-7267.

38 T. Mutai, H. Sawatani, T. Shida, H. Shono and K. Araki, J. Org. Chem., 2013, 78, 2482-2489.

39 N. Zhao, Y. Li, Y. Jia and P. Li, J. Phys. Chem. C, 2018, 122, 26576-26583.

40 A. P. Demchenko, K. C. Tang and P. T. Chou, Chem. Soc. Rev., 2013, 42, 1379-1408.

41 L. Chen, J.-W. Ye, H.-P. Wang, M. Pan, S.-Y. Yin, Z.-W. Wei, L.-Y. Zhang, K. Wu, Y.-N. Fan and C.-Y. Su, Nat. Commun., 2017, 8, 15985.

42 A. Douvali, A. C. Tsipis, S. V. Eliseeva, S. Petoud, G. S. Papaefstathiou, C. D. Malliakas, I. Papadas, G. S. Armatas, I. Margiolaki, M. G. Kanatzidis, T. Lazarides and M. J. Manos, Angew. Chem., Int. Ed., 2015, 54, 1651-1656.

43 J. Zhao, S. Ji, Y. Chen, H. Guo and P. Yang, Phys. Chem. Chem. Phys., 2012, 14, 8803-8817.

44 O. M. Zamotaiev, V. Y. Postupalenko, V. V. Shvadchak, V. G. Pivovarenko, A. S. Klymchenko and Y. Mély, Bioconjugate Chem., 2011, 22, 101-107. 